\title{
On-sky testing of algorithms for extended LGS spots
}

\author{
Alastair Basden, ${ }^{a}$ Andrew Reeves, Lisa Bardou, Domenico Bonaccini Calia, Tristan Buey, \\ Mauro Centrone, Fanny Chemla, Philippe Feautrier, Jean-Luc Gach, Eric Gendron, Damien \\ Gratadour, David Jenkins, Gianluca Lombardi, Enrico Marchetti, Tim Morris, Richard Myers, \\ James Osborn, Thomas Pfrommer, Marcos Reyes Garcia Talavera, Gerard Rousset, Eric \\ Stadler, Robert Talbot, Matthew Townson, Fabrice Vidal \\ ${ }^{a}$ Department of Phyics, Durham University, South Road, Durham, DH1 3LE, UK.
}

\begin{abstract}
We present results of on-sky testing of different algorithms for highly extended LGS spots. We have used the CANARY wide-field AO demonstrator instrument on the $4.2 \mathrm{~m}$ William Herschel Telescope on La Palma with the ESO Wendelstein LGS unit, launched 40m off-axis to simulate edge sub-apertures of the E-ELT. We investigate the performance of correlation and matched filter algorithms, comparing performance with a traditional centre of gravity. We also present on-sky tests of advanced pixel handling algorithms which we show improve AO system performance, including adaptive windowing (spot tracking), total variation minimisation and brightest pixel selection. Techniques to improve detection of truncated spots are also presented.
\end{abstract}

Additionally, we present a critical study of Shack-Hartmann sensor accuracy with accurate detector readout models, highly applicable to AO system designers when specifying detector performance, or for predicting $\mathrm{AO}$ system performance with a given detector.

\section{INTRODUCTION}

When adaptive optics (AO) systems are used to improve the effective resolution of astronomical images, sky coverage becomes limited by the availability of sufficiently bright natural guide stars (NGSs). The use of laser guide stars (LGSs) can significantly improve sky coverage, though at least one NGS is still required. LGSs introduce an additional complexity, and in particular, unlike a NGS, the structure of a LGS is not a point source. Rather, the LGSs will appear as elongated structures due to the perspective effect of viewing a line extended object off-axis, as shown in Fig. 1.

This elongation is particularly pronounced for Extremely Large Telescopes (ELTs), where the edge subapertures of a Shack-Hartmann wavefront sensor (WFS) can be a large distance from the laser launch location, and therefore view the LGS probe far off axis. When the LGSs are launched from behind the telescope secondary mirror, as will be the case for the Thirty Metre Telescope (TMT), the maximum off-axis distance is approximately equal to the telescope radius, or $15 \mathrm{~m}$ in the case of TMT. However, if the LGSs are launched from the side of the aperture, as is the case for the European Southern Observatory (ESO) ELT, the maximum off-axis sub-aperture distance will be greater than the telescope diameter, or about $40 \mathrm{~m}$ in this case. Fig. 2 demonstrates this effect.

As LGS signal becomes more elongated for sub-apertures further from the launch location, the available photon flux will be spread across a greater number of sub-apertures. Therefore, signal to noise ratio (SNR) will be reduced and the accuracy of local wavefront gradient estimation will decrease. It is interesting to note, as demonstrated by Fig. 2(a), that even viewing an ELT WFS image will be problematic, due to the large dynamic range required. It would be expected that ELT software (real-time image displays) should be able to offer several different viewing modes and palettes to provide AO scientists with improved system understanding.

Further author information: (Send correspondence to Alastair Basden)

Alastair Basden: E-mail: a.g.basden.97 (a t) cantab.net, Telephone: +44 1913342229 


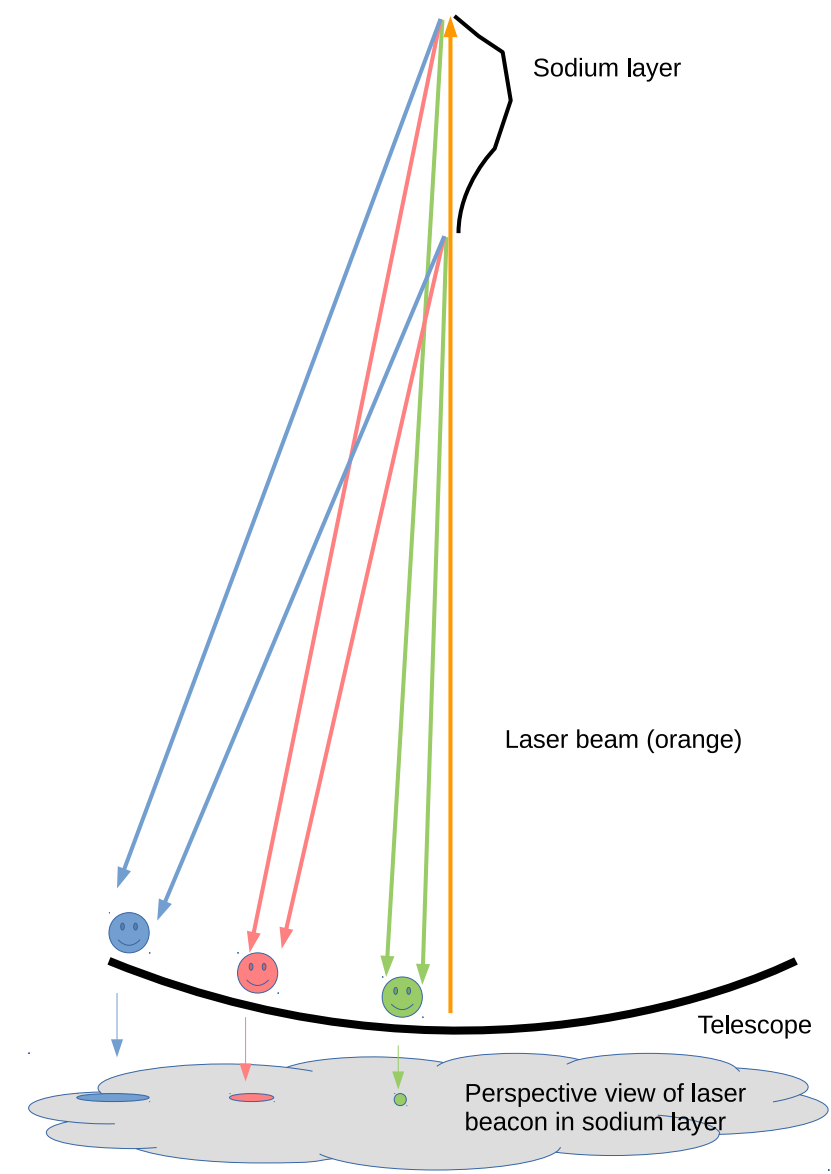

Figure 1. A figure demonstrating the elongation of LGSs. A LGS is formed either from Rayleigh back scattering of a laser beam as it travels through the atmosphere, or through interaction with sodium atoms in the mesosphere. This results in an elongated structure, which when viewed at greater distances off axis will result in an increasing perceived elongation as shown.
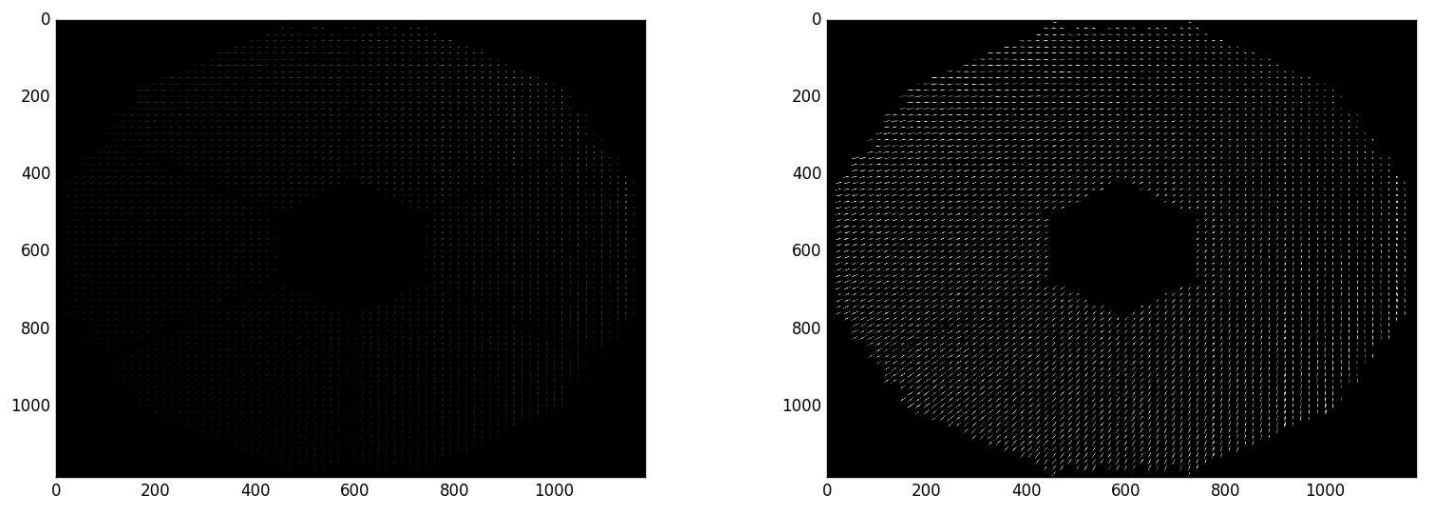

Figure 2. If you are viewing this image on a computer screen, it may be necessary to zoom in. (a) A Shack-Hartmann LGS wavefront sensor image for the ELT. It is evident that the more elongated sub-apertures are more difficult to see since flux is spread over a greater number of pixels. (b) As for (a), but with the maximum intensity of each sub-aperture normalised to unity. 


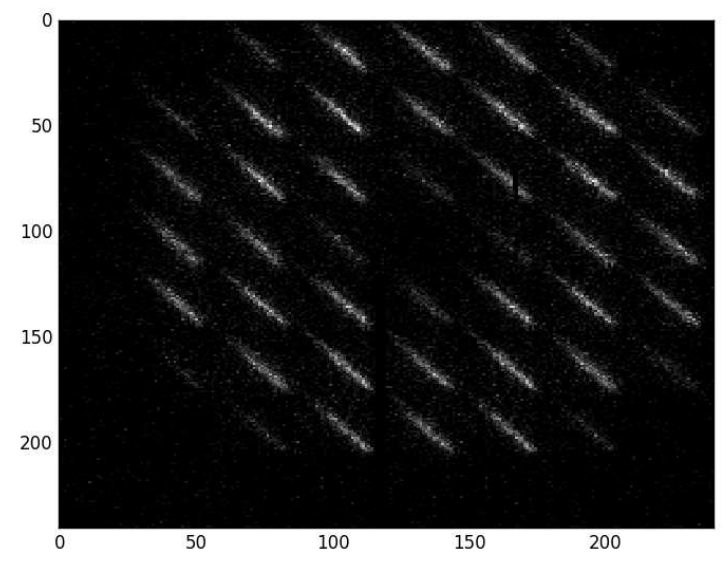

Figure 3. A single LGS WFS raw image frame from CANARY phase D.

\subsection{The CANARY AO demonstrator}

The CANARY instrument ${ }^{1}$ on the William Herschel Telescope (WHT) is an AO technology demonstrator, which has, over the past decade, provided on-sky demonstration of many technologies critical to successful ELT operation, including multi-object AO (MOAO) ${ }^{2}$ laser tomographic AO (LTAO), ${ }^{3}$ CPU-based real-time control for wide-field AO, ${ }^{4} \mathrm{GPU}$ acceleration techniques, ${ }^{5}$ advanced sub-aperture processing algorithms, ${ }^{6-8}$ pseudo-openloop control, integrated use of deformable mirror (DM) figure sensors, and many other techniques. CANARY has also yielded a vast wealth of experience with the use of multiple wavefront sensors of different types, and multiple DMs. ${ }^{9}$

The phase D configuration of CANARY sees the use of a sodium LGS launched about $40 \mathrm{~m}$ from the telescope axis. The LGS is viewed by a $7 \times 7$ sub-aperture Shack-Hartmann WFS. Additionally, CANARY has 3 off-axis NGS, and an on-axis truth sensor, all with $7 \times 7$ sub-apertures. A single DM, with $8 \times 8$ actuators (52 active), is used in closed loop (i.e. the WFSs are sensitive to changes in DM shape).

The large off-axis launch distance for the LGS means that all WFS sub-apertures see a highly elongated structure, as shown in Fig. 3. This is by design, and allows this WFS to mimic a sub-pupil at the edge of the ELT. Therefore, CANARY provides an ideal way of investigating algorithms to aid the processing of these elongated structures. In particular, the LGS and on-axis NGS are co-located, and so view the same atmospheric turbulence (focal anisoplanatism is small for a sodium LGS on a $4 \mathrm{~m}$ telescope). We can therefore use NGS measurements as a truth value when investigating the performance of different LGS algorithms. During CANARY operation, large open-loop datasets have been captured, so that the performance of new and novel algorithms can be explored without needing on-sky operation. Some of these algorithms are also tested on-sky with the AO loop engaged.

In $\S 2$, we explore the algorithms that we have investigated to help mitigate LGS elongation. Additional novel approaches are explored in $\S 3$, and we conclude in $\S 4$.

\section{EXPLORATION OF LGS ELONGATION MITIGATION}

The CANARY LGS WFS uses $30 \times 30$ pixels per sub-aperture. This provides the necessary flexibility to explore different options for the ELT. The ELT will have $80 \times 80$ sub-apertures for each LGS WFS. If these are to each have $30 \times 30$ pixels, then the volume of data (requiring transmission and processing) would be huge at AO frame rates (e.g. nearly $100 \mathrm{GBit} / \mathrm{s}$ at $1 \mathrm{kHz}$ ). However, using CANARY data, we can explore the trade-off between number of pixels, field of view and pixel scale. It is evident that increased numbers of pixels results in increased noise, since each pixel introduces an associated readout noise. 

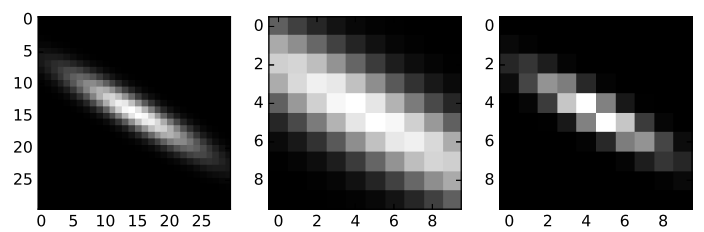

Figure 4. (a) An example CANARY LGS Shack-Hartmann spot. (b) As for (a), truncated to $10 \times 10$ pixels. (c) As for (a), with the pixel scale increased so that the spot fits within $10 \times 10$ pixels without truncation.

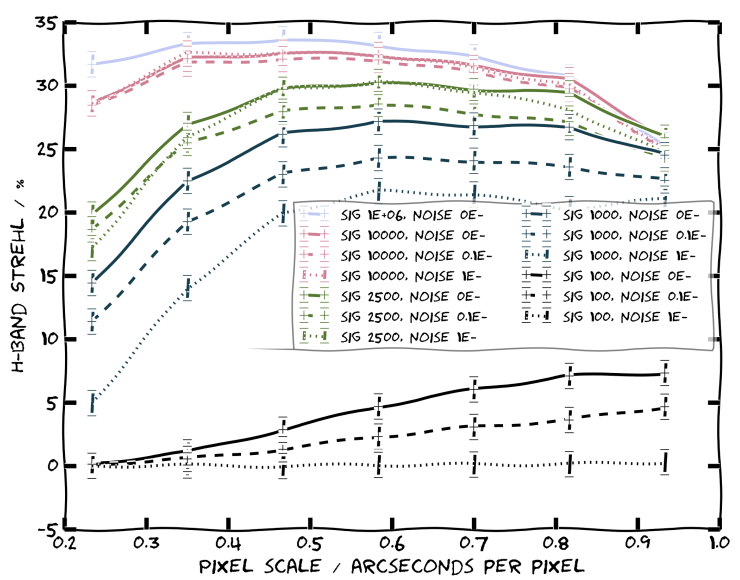

Figure 5. A figure showing AO performance (H-band Strelh ration) for a wide-field ELT AO system as a function of pixel scale. Cases with different numbers of pixels are shown, as given in the legend.

Suitable detectors for the ELT include the LVSM which is under development by ESO and Teledyne-E2V, and a Fairchild Imaging LTN4625A detector. Both detectors are CMOS. The LVSM has $840 \times 840$ pixels, allowing $10 \times 10$ pixels per sub-aperture, and can achieve frame rates of up to $1 \mathrm{kHz}$. The pixels are large, an important consideration for precise alignment of ELT AO systems. The LTN4625A detector has $4608 \times 2592$ pixels, which would allow $30 \times 30$ pixels per sub-aperture. However, maximum frame rate is restricted to $240 \mathrm{~Hz}$, which is not fast enough for some ELT instruments. The pixels are small, meaning that optical alignment would be more involved. We therefore concentrate here on the LVSM detector.

When moving from $30 \times 30$ pixels available to CANARY to the $10 \times 10$ pixels of the LVSM, we have three options. Either the LGS spot images can be truncated. Alternatively, the pixel scale can be increased, squashing the spot pattern into fewer pixels, and reducing sensitivity. Finally, a combination of these approaches can be taken. Fig. 4 shows a CANARY spot, a truncated version and a compressed version for comparison. The approach taken will have some impact on AO performance, as shown in Fig. 5. Were, we can see that pixel scales between $0.6-0.8 \mathrm{arcsec} /$ pixel provide best performance, and that intermediate sub-aperture sizes $($ e.g. $10 \times 10$ pixels) can provide reasonable results. Further results are given by. ${ }^{10,11}$

\subsection{On-sky testing of WFS processing algorithms}

\subsubsection{Correlation}

CANARY can use a FFT based correlation algorithm, with zero padding to avoid bias. Computation of local wavefront gradients is performed as follows:

1. A correlation reference image is obtained, using the average of many frames of WFS images (typically 100, shifted and added). A different reference image is used for each sub-aperture. 

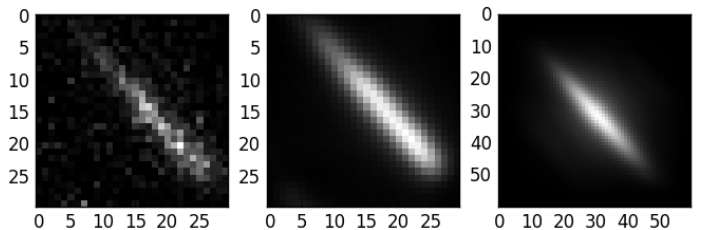

Figure 6. (a) A raw LGS image. (b) Reference correlation image, using 100 frames shifted and added. (c) The resulting correlation signal when correlating (a) with (b).

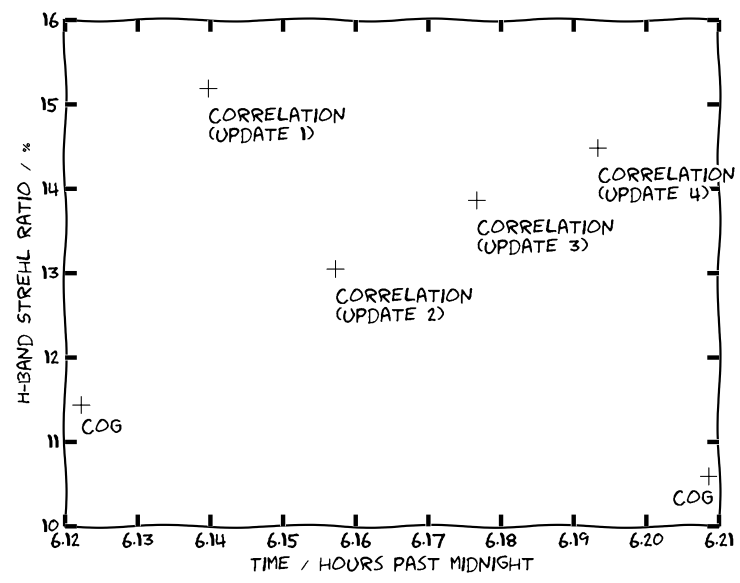

Figure 7. On-sky AO performance with CANARY, showing performance using both CoG and correlation. Between correlation points, the correlation reference images and slopes were updated while the AO loop was engaged.

2. The correlation reference is zero padded, to at least twice the size, and the FFT computed (stored in half-complex format).

3. For each iteration, and each sub-aperture, the Shack-Hartmann sub-aperture images are zero-padded and the FFT computed.

4. The image FFT is multiplied by the reference FFT, and the inverse FFT computed. This results in the correlation image

5. A threshold is applied to the correlation image, at some fraction of the peak intensity.

6. A centre of gravity algorithm is then used to compute the spot position, and hence local wavefront gradient.

7. Reference slope measurements are subtracted.

Fig. 6 shows a typical sub-aperture image, a reference image, and the resulting correlation. It is important to note, as described by Basden et $a{ }^{7}{ }^{7}$ that reference slopes must be updated to match the reference image whenever the reference image is changed. Otherwise, although a stable AO corrected point spread function (PSF) will result, it will not be optimal, and will change whenever the reference image is changed. Fig. 7 shows some on-sky results, with further details given by Basden et al. ${ }^{7}$

\subsubsection{Automatic reference image update}

The Durham AO real-time controller (DARC) ${ }^{4}$ used by CANARY has the ability to continuously update correlation reference images and corresponding reference slopes. Here, at every AO time-step, a rolling shift-add 

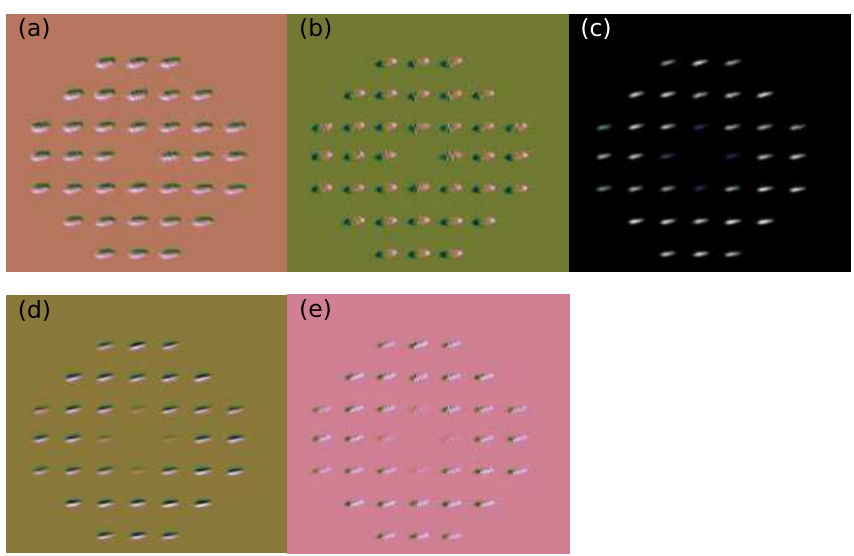

Figure 8. A figure showing the components of a matched filter for the CANARY LGS WFS. (a) and (b) show the image gradients in $\mathrm{x}$ and $\mathrm{y}$. (c) shows the averaged reference image, while (d) and (e) show the $\mathrm{x}$ and $\mathrm{y}$ components of the matched filter.

average of sub-aperture images is computed, leading to the correlation reference, and new reference slopes (updated by an amount equal to the centre of gravity of the result of the correlation of current and previous reference image). This is ideal for use when the structure of the elongated image is changing e.g. a meteor event within the mesosphere changing the structure of the sodium layer. However computational load is high, so DARC has the ability to update reference images (and slopes) on a rolling basis, for example, spreading the update over 10 iterations (i.e. updating a tenth of reference images every frame)

We note that this automatic refernce image updating technique can yield a large amount of telemetry information for diagnosis, including the reference images, the reference slopes, and the current correlation pattern.

\subsubsection{Matched filtering}

A noise optimal technique for wavefront slope estimation has been proposed, ${ }^{12}$ and has been selected for use with TMT. Computationally, within the real-time control system, a matched filter technique is efficient, requiring only slightly more computational power than a centre of gravity. Computation of the matched filter itself is more demanding, though this is not necessary in real-time, but can be performed away from the real-time control system.

Computation of the matched filter requires a measurement of sub-aperture image gradients. This is achieved by on-sky dithering of the LGS spot, and is dependent on both atmospheric conditions and laser guide star image structure. Therefore, this computation is an ongoing process. The matched filtering technique is also non-linear, with the estimated slope measurement not corresponding directly with the actual wavefront slope. We use a range extension algorithm ${ }^{13}$ which is able to extend linearity to approximately one pixel. We then use active spot tracking (adaptive windowing) to further mitigate this problem: the centre of a sub-aperture is defined from the spot measurement in the previous frame.

We use a separate matched filter for each sub-aperture, and also have the ability to have different sized sub-apertures.

We note that due to the non-linearity of the matched filter algorithm, we are not able to compute reference slope measurements based on previous measurements. Rather, they must be updated on-sky, i.e. open loop measurements must first be taken to average reference slopes before a new matched filter can be used.

Fig. 8 shows the components of a matched filter, including the measured imagr gradients ( $\mathrm{x}$ and $\mathrm{y}$ ), the averaged image, and the matched filter itself. Fig. 9 shows on-sky results obtained using the matched filter algorithm. Further results and details are given by Basden et $\mathrm{al}^{14}$. 


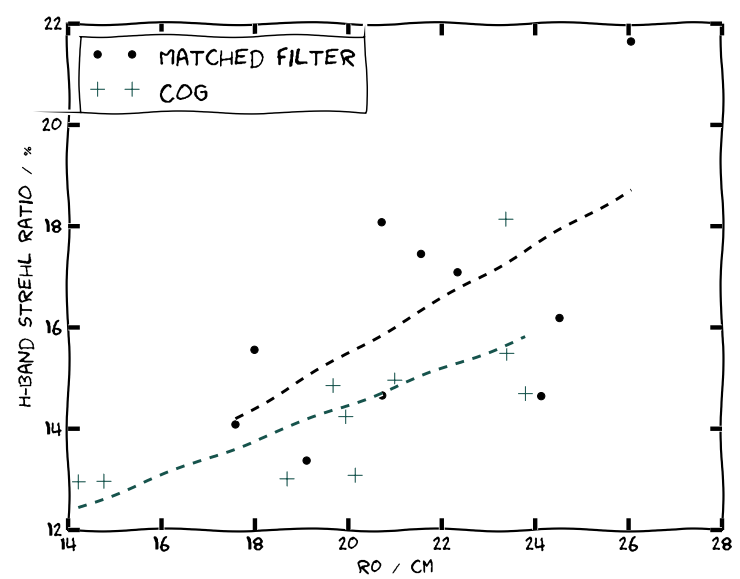

Figure 9. A figure showing AO performance (H-band Strehl ratio) using CANARY as a function of seeing strength. Results comparing the matched filter and centre of gravity techniques are shown, as given in the legend.

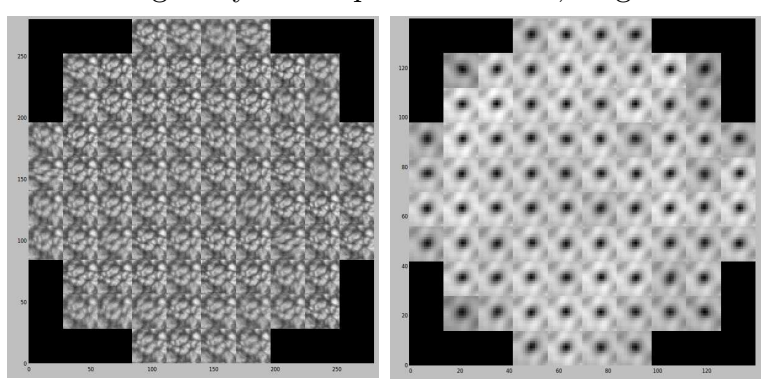

Figure 10. (a) A representative solar wavefront sensor image. (b) The corresponding correlation signal, computed using difference squared correlation. The minima within each sub-aperture represent the local wavefront gradient.

\subsubsection{Difference squared correlation}

With solar AO systems, best performance for wavefront gradient estimation has been shown to be achieved using a difference-squared correlation technique. Here, the sum of the square difference between the reference and instantaneous wavefront sensor images are computed as a function of $\mathrm{x}$ and $\mathrm{y}$ offest between the image and reference. This results in a trough in the resulting correlation where the image and reference are best aligned. A parabolic fit can then be used to determine the sub-pixel position of this trough, and hence the local wavefront gradient. Fig. 10 shows an example solar wavefront image, and corresponding correlation signal, computed using the Durham AO simulation platform (DASP). ${ }^{15}$

For LGS images, we find that difference-squared correlation is not so good, and research is ongoing. However, it has the potential to offer an ideal technique for handing spot truncation. Fig. 11 shows a representitive difference squared correlation signal using CANARY data.

\subsubsection{Spot truncation mitigation}

When local wavefront gradients are large, the LGS spots can move partially outside their assigned sub-aperture, and therefore truncation of the spot occurs. This is problematic. If a centre of gravity algorithm is used, the slope estimate becomes biased (since this algorithm cannot compute slopes larger than the sub-aperture size). If a correlation algorithm is used, the truncated image has a different format and structure than the reference image, and so the correlation signal becomes weaker, particularly when FFT-based correlation is used. In this case, a truncated refernce can also be used, i.e. a two-step process, with crude spot position being estimated, the reference image truncated at this position, and then used to compute the correlation. However this is 


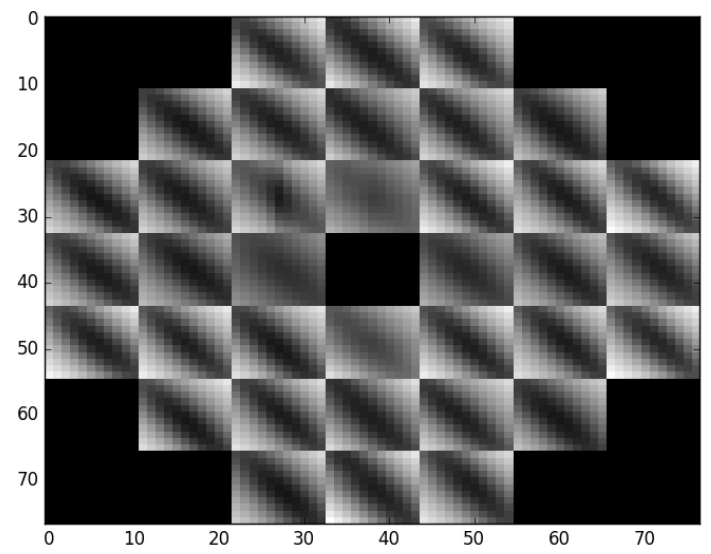

Figure 11. A representative difference squared correlation image using a CANARY WFS image.

computationally expensive and requires fine parameter tuning, so is not robust. The use of over-sized references are also challenging.

Difference-squared correlation is also a promising technique here, though work is ongoing.

\subsection{Detector modelling}

Current AO systems generally use CCD detectors. However, it is likely that the ELT WFSs will use CMOS detectors. These have individual pixel readout, and therefore each pixel in a CMOS detector has its own characteristic readout noise level. Such detectors are often supplied with a quoted median root-mean-square (RMS) noise level. Unfortunately, the distribution of readout noise levels has a large tail, and so some pixels can have a significantly larger readout noise, which can affect the WFS image. Basden et $\mathrm{al}^{16}$ provide an in-depth study of this effect, and Fig. 12 shows estimated wavefront slope error as a function of sub-aperture size for different readout noise models. These models include a uniform readout noise level (i.e. the same readout noise for each pixel) based on the manufacturer quoted median and mean values, and also a full stochastic readout noise level, where each pixel has a different readout noise level (though with the same median and mean as the other models). It can be seen that for larger sub-aperture sizes, this has a significant impact on performance.

\section{IMAGE CALIBRATION APPROACHES AND OTHER CONCEPTS}

With CANARY, we have investigated, and regularly use other image calibration approaches to improve AO performance.

\subsection{Spot tracking}

Spot tracking, or adaptive windowing, is a technique that allows sub-aperture positions to follow the spots within them, based on the spot location during previous frames. This technique allows us to reduce sub-aperture size, while still maintaining field of view. The reduction in sub-aperture size is beneficial because less readout noise is introduced. Spot tracking is used routinely with CANARY.

When using this technique, some additional considerations need to be taken into account. The sub-apertures should not be allowed to wander arbitrarily large amounts, as otherwise, they may latch on to neighbouring spots. Additionally, if a sub-aperture becomes stuck at one limit of its motion for a specified number of WFS frames, it should automatically be reset to the centre of the sub-aperture. This is to avoid effects such as sky brightness gradients (particularly near dusk and dawn) impacting AO performance. 


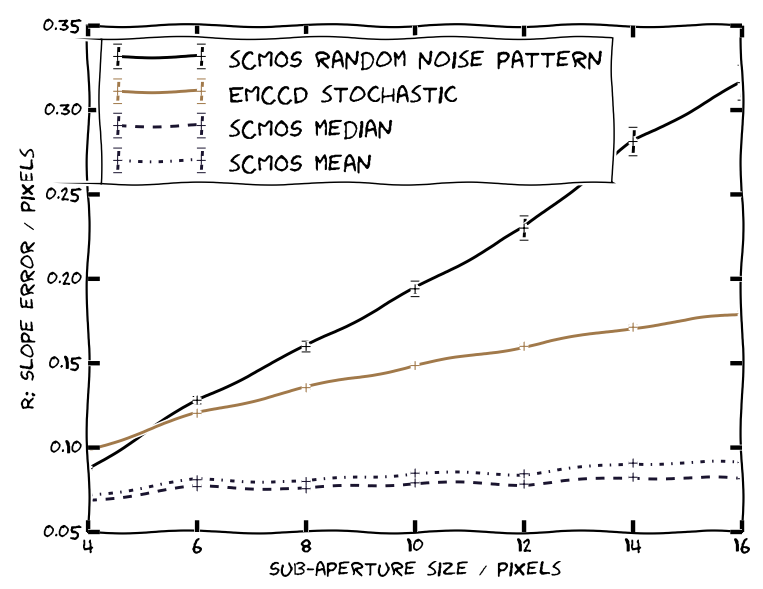

Figure 12. A figure showing slope estimation error (larger is worse) as a function of sub-aperture edge size for different CMOS noise models. Comparison with an EMCCD is also shown.
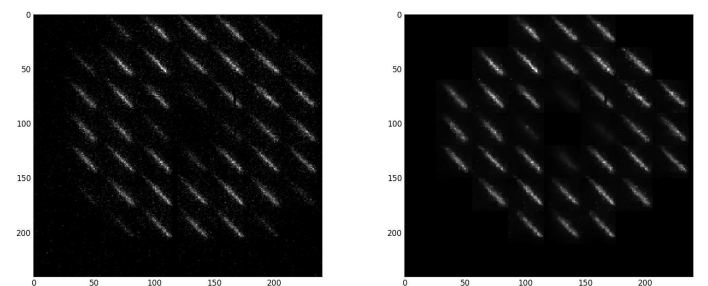

Figure 13. (a) A raw LGS image from CANARY. (b) The LGS image after application of TVM.

\subsection{Brightest pixel selection}

A Shack-Hartmann sub-aperture has a well know form: it will contain a spot of a particular area, and noise. By limiting the number of pixels used in spot position estimation, performance improvements can be achieved since pixels containing only readout noise are ignored. This technique effectively computes a background threshold level on a frame-by-frame and sub-aperture-by-sub-aperture basis. Investigations into performance improvements have previously been carried out, ${ }^{6}$ so we do not repeat this here.

\subsection{Arbitrary sub-aperture shapes}

The shape of an LGS spot will be well known (determinable from the wavefront sensor image data). Therefore, by applying a sub-aperture shape that is will matched to this shape, the number of pixels in each sub-aperture can be significantly reduced, and performance increased. Since LGSs are elongated, a rectangular or oval subaperture is most appropriate, rotated to align with the elongation. The CANARY real-time control system (RTCS) has the ability to use this technique.

\subsection{Total variation minimisation}

Total variation minimisation ${ }^{8}$ is an image processing technique which aims to reduce noise within an image by minmising the total image variance. This approach is good at leaving image features intact, and therefore offers potential for AO improvements. Fig. 13 shows a raw image, and one cleaned using total variation minimisation (TVM), and it is clear that the signal is more evident within the cleaned image. On-sky testing of this technique has shown that AO performance can be improved. ${ }^{8}$ 


\subsection{Astigmatic lenslet arrays}

An optical approach that can be used to mitigate some of the elongation issues associated with LGSs is to use astigmatic lenslet arrays. Here, the elongated spot can be compressed along the elongation direction, reducing the number of pixels required (and hence readout noise), and increasing the peak intensity of the image. Unfortunately, sensitivity (pixel scale) is also reduced in the compressed dimension, and so algorithms are required to take into account the different sensitivities of the slope measurement along, and perpendicular to the elongation direction.

\subsection{Variable pixel scale LGS WFS}

If the lenslet array focal length is varied across the sensor, and the focal plane detector tilted, a variable sampling system can be obtained. This allows spots close to the launch axis to be well sampled with high sensitivity, while spots further from the launch axis can avoid truncation (for a given number of detector pixels), focus light into fewer pixels, but have reduced sensitivity.

\subsection{Variable sampling LGS WFS}

Another alternative optical arrangement is to alter the size of sub-apertures across the sensor. Close to the launch axis, sub-aperture sizes can be small, with few pixels per sub-aperture. Further from the launch axis, the sub-aperture size can be increased so that more light is collected. The spots will still be highly elongated, but will have greater signal, and thus AO performance can be improved.

\subsection{High altitude spatially stabilised light sources}

LGSs do not fully overcome the sky coverage problem, since NGSs are still required for global wavefront tilt measurement. However, by using a light source on a unmanned aerial vehicle (UAV) platform, combined with precise position knowledge derived from accelerometer, gyroscope and global navigation satellite system signals, there is the potential to remove the NGS requirement, and thus achieve full sky coverage. This is explored further by Basden et $\mathrm{al}^{17}$

\section{CONCLUSIONS}

We have explored techniques to aid the processing of elongated LGS spot patterns, and identified methods to improve AO performance. On-sky testing with the CANARY AO demonstrator has verified these techniques, and quantified the possible improvements. We have also discussed additional non-standard optical designs for lenslet arrays which can help to mitigate the elongation problem. However, although these techniques lead to demonstrable performance gains, we predict that the ELTs will actually end up using centre of gravity algorithms for wavefront gradient estimation. Commissioning time on the ELTs will be severely restricted, and there will be many major problems to overcome, before moving on to slight performance improvements. The robustness and simplicity of the centre of gravity will outweigh any performance gains, at least initially (in the current opinion of the lead author!).

\section{REFERENCES}

[1] Myers, R. M., Hubert, Z., Morris, T. J., Gendron, E., Dipper, N. A., Kellerer, A., Goodsell, S. J., Rousset, G., Younger, E., Marteaud, M., Basden, A. G., Chemla, F., Guzman, C. D., Fusco, T., Geng, D., Le Roux, B., Harrison, M. A., Longmore, A. J., Young, L. K., Vidal, F., and Greenaway, A. H., "CANARY: the onsky NGS/LGS MOAO demonstrator for EAGLE," in [Society of Photo-Optical Instrumentation Engineers (SPIE) Conference Series], Society of Photo-Optical Instrumentation Engineers (SPIE) Conference Series 7015, 0 (July 2008).

[2] Gendron, E., Vidal, F., Brangier, M., Morris, T., Hubert, Z., Basden, A. G., Rousset, G., Myers, R., Chemla, F., Longmore, A., Butterley, T., Dipper, N., Dunlop, C., Geng, D., Gratadour, D., Henry, D., Laporte, P., Looker, N., Perret, D., Sevin, A., Talbot, G., and Younger, E., "MOAO first on-sky demonstration with CANARY," A $B A$ 529, L2 (May 2011). 
[3] Le Louarn, M., Clare, R., Bchet, C., and Tallon, M., "Simulations of adaptive optics systems for the eelt," in [Society of Photo-Optical Instrumentation Engineers (SPIE) Conference Series], Proc. SPIE 8447, 84475D-84475D-7 (July 2012).

[4] Basden, A. G., Geng, D., Myers, R., and Younger, E., "Durham adaptive optics real-time controller," Appl. Optics 49, 6354-6363 (Nov. 2010).

[5] Basden, A. G. and Myers, R. M., "The Durham adaptive optics real-time controller: capability and Extremely Large Telescope suitability," MNRAS 424, 1483-1494 (Aug. 2012).

[6] Basden, A. G., Myers, R. M., and Gendron, E., "Wavefront sensing with a brightest pixel selection algorithm," MNRAS 419, 1628-1636 (Jan. 2012).

[7] Basden, A. G., Chemla, F., Dipper, N., Gendron, E., Henry, D., Morris, T., Rousset, G., and Vidal, F., "Real-time correlation reference update for astronomical adaptive optics," MNRAS 439, 968-976 (Mar. 2014).

[8] Basden, A. G., Morris, T. J., Gratadour, D., and Gendron, E., "Sensitivity improvements for ShackHartmann wavefront sensors using total variation minimization," MNRAS 449, 3537-3542 (June 2015).

[9] Basden, A. G., Atkinson, D., Bharmal, N. A., Bitenc, U., Brangier, M., Buey, T., Butterley, T., Cano, D., Chemla, F., Clark, P., Cohen, M., Conan, J.-M., de Cos, F. J., Dickson, C., Dipper, N. A., Dunlop, C. N., Feautrier, P., Fusco, T., Gach, J. L., Gendron, E., Geng, D., Goodsell, S. J., Gratadour, D., Greenaway, A. H., Guesalaga, A., Guzman, C. D., Henry, D., Holck, D., Hubert, Z., Huet, J. M., Kellerer, A., Kulcsar, C., Laporte, P., Le Roux, B., Looker, N., Longmore, A. J., Marteaud, M., Martin, O., Meimon, S., Morel, C., Morris, T. J., Myers, R. M., Osborn, J., Perret, D., Petit, C., Raynaud, H., Reeves, A. P., Rousset, G., Sanchez Lasheras, F., Sanchez Rodriguez, M., Santos, J. D., Sevin, A., Sivo, G., Stadler, E., Stobie, B., Talbot, G., Todd, S., Vidal, F., and Younger, E. J., "Experience with wavefront sensor and deformable mirror interfaces for wide-field adaptive optics systems," MNRAS 459, 1350-1359 (June 2016).

[10] Basden, A. G., "Monte Carlo modelling of multiconjugate adaptive optics performance on the European Extremely Large Telescope," MNRAS 453, 3035-3042 (Nov. 2015).

[11] Basden, A. G. and Morris, T. J., "Monte Carlo modelling of multi-object adaptive optics performance on the European Extremely Large Telescope," MNRAS 463, 4184-4193 (Dec. 2016).

[12] Gilles, L., Ellerbroek, B., and Véran, J.-P., "Laser guide star multi-conjugate adaptive optics performance of the Thirty Meter Telescope with elongated beacons and matched filtering," in [Society of Photo-Optical Instrumentation Engineers (SPIE) Conference Series], Society of Photo-Optical Instrumentation Engineers (SPIE) Conference Series 6272, 627236 (June 2006).

[13] Gilles, L. and Ellerbroek, B. L., "Constrained matched filtering for extended dynamic range and improved noise rejection for Shack-Hartmann wavefront sensing," Optics Letters 33, 1159 (May 2008).

[14] Basden, A. G., Bardou, L., Bonaccini Calia, D., Buey, T., Centrone, M., Chemla, F., Gach, J. L., Gendron, E., Gratadour, D., Guidolin, I., Jenkins, D. R., Marchetti, E., Morris, T. J., Myers, R. M., Osborn, J., Reeves, A. P., Reyes, M., Rousset, G., Lombardi, G., Townson, M. J., and Vidal, F., "On-sky demonstration of matched filters for wavefront measurements using ELT-scale elongated laser guide stars," MNRAS 466, 5003-5010 (Apr. 2017).

[15] Basden, A. G., Butterley, T., Myers, R. M., and Wilson, R. W., "Durham extremely large telescope adaptive optics simulation platform," Appl. Optics 46, 1089-1098 (Mar. 2007).

[16] Basden, A. G., "Analysis of electron multiplying charge coupled device and scientific CMOS readout noise models for ShackHartmann wavefront sensor accuracy," JATIS 1(3), 039002-1 - 039002-10 (July 2015).

[17] Basden, A. G., Brown, A., Chadwick, P., Clark, P., and Massey, R. J., "Artificial guide stars for adaptive optics using unmanned aerial vehicles," In prep. (2017). 